\title{
Mapping the Extended H I Distribution of Three Dwarf Galaxies
}

\author{
Deidre A. Hunter, Fakhri Zahedy, Emily C. Bowsher ${ }^{1} 3$ \\ Lowell Observatory, 1400 West Mars Hill Road, Flagstaff, Arizona 86001 USA \\ dah@lowell.edu, fsz@mit.edu, bowsher@chara.gsu.edu \\ Eric M. Wilcots, ${ }^{1}$ Amanda A. Kepley ${ }^{1}{ }^{4}$ and Veronika Gaal ${ }^{1}$ \\ Department of Astronomy, University of Wisconsin, 475 N. Charter St., Madison, \\ Wisconsin 53706-1582 USA \\ ewilcots@astro.wisc.edu, aak8t@mail.astro.virginia.edu
}

\begin{abstract}
We present large field $\mathrm{H}$ I-line emission maps obtained with the single-dish Green Bank Telescope centered on the dwarf irregular galaxies Sextans A, NGC 2366, and WLM. We do not detect the extended skirts of emission associated with the galaxies that were reported from Effelsberg observations (Huchtmeier et al. 1981). The ratio of $\mathrm{H}$ I at $10^{19}$ atoms $\mathrm{cm}^{-2}$ to optical extents of these galaxies are instead 2-3, which is normal for this type of galaxy. There is no evidence for a truncation in the $\mathrm{H}$ I distribution $\geq 10^{19}$ atoms $\mathrm{cm}^{-2}$.
\end{abstract}

Subject headings: galaxies: irregular — galaxies: individual (Sextans A,NGC 2366, WLM) — galaxies: ISM

\footnotetext{
${ }^{1}$ Guest Observer, Green Bank Telescope, National Radio Astronomy Observatory, a facility of the National Science Foundation operated under cooperative agreement by Associated Universities, Inc.

${ }^{2}$ Current address: Department of Physics, MIT, 77 Massachusetts Avenue, Cambridge, Massachusetts $02139-4307$

${ }^{3}$ Current address: Center for Astrophysics and Space Sciences, University of California San Diego, 9500 Gilman Drive, La Jolla, California 92093-0354 and Columbia Astrophysics Laboratory, Columbia University, Mail Code 5247, 550 West 120th Street, New York, New York 10027

${ }^{4}$ Current address: Department of Astronomy, University of Virginia, Charlottesville, Virginia 22904-4325
} 


\section{Introduction}

Dwarf irregular (dIm) galaxies, like many disk systems, often have neutral atomic hydrogen gas that extends beyond, and sometimes well beyond, the optical galaxy. In extreme cases this gas is known to extend 3-7 times farther than the stellar distributions (Huchtmeier et al. 1981, Bajaja et al. 1994). Such envelopes were believed to be relatively quiescent reservoirs of gas, as there was little data to the contrary. This changed with Very Large Array $\left(\mathrm{VLA}^{5}\right.$ ) mosaics of NGC 4449 (Hunter et al. 1998) and IC 10 (Shostak \& Skillman 1989, Wilcots \& Miller 1999). NGC 4449 is surrounded by a complex network of streamers that represents the remains of an $\mathrm{H}$ I disk disrupted by an encounter with another galaxy (Hunter et al. 1998, Theis \& Kohle 1998). IC 10 is embedded in a counter-rotating halo of H I and also appears to be currently accreting a large cloud of gas (Wilcots \& Miller 1998). On the other hand, some dwarfs are embedded in large, quiescent disks that are simply smooth extensions of the galaxy that happen to stretch some 5-7 times farther than the stars (Carignan \& Purton 1998; Kreckel et al. 2011).

We do not yet understand how some irregulars have maintained extremely large quiescent disks, especially given the onslaught of activity and ionization in the early universe (Ricotti et al. 2001). Similarly, it is not yet clear what role the extended gas around irregulars plays in the evolution of the stellar disk. Also, the extended gas is more sensitive to interactions with other galaxies. Therefore, it is of interest to determine the full extent of H I distributions around galaxies.

One galaxy with a potentially large smooth extension of gas is the dwarf irregular Sextans A. Single dish maps of Sextans A by Huchtmeier et al. (1981) with the Effelsberg $100 \mathrm{~m}$ telescope showed $\mathrm{H}$ I extending to a diameter of $54^{\prime}$ at a column density of $10^{19}$ atoms $\mathrm{cm}^{-2}$. Thus, H I was detected to 8.7 times the galaxy's optically-defined Holmberg diameter. This motivated Wilcots \& Hunter (2002) to map Sextans A in H I using the VLA in a mosaic that covered $90^{\prime}$ diameter at $1^{\prime}$ resolution (see also, Barnes \& De Blok 2004). Although those observations reached a column density of $7.5 \times 10^{18}$ atoms $\mathrm{cm}^{-2}$, H I was only detected to a diameter of $18^{\prime}$, about one-third of the extent reported by Huchtmeier et al., and only about $62 \%$ of the total H I flux reported by Huchtmeier et al. was recovered. Wilcots and Hunter suggested that the missing gas exists in a very smooth, low column density distribution with a size scale too large to be resolved with the interferometer. They estimated that the density fluctuations in this extended gas should be no smaller than $10^{\prime}-15^{\prime}$ to be hidden from the VLA.

\footnotetext{
${ }^{5}$ The VLA and GBT are facilities of the National Radio Astronomy Observatory. NRAO is a facility of the National Science Foundation operated under cooperative agreement by Associated Universities, Inc.
} 
If the extended gas around Sextans $\mathrm{A}$ is indeed that smooth, it is a prime target for mapping with the Green Bank Telescope $\left(G^{5} T^{5}\right)$. First, because of its unique design, the GBT has extraordinarily low side lobes: $30 \mathrm{~dB}$ below the primary beam at $1420 \mathrm{MHz}$, about $0.1 \%$. This allows one to detect faint gas far from the center of the galaxy. Second, with a beam-size at 21-cm of $8.7^{\prime}$ the GBT would still be able to resolve large-scale structure in the extended gas if it exists. We, therefore, undertook to use the GBT to map the H I gas around Sextans A. Our purpose was to use the unparalleled sensitivity of GBT to confirm the existence of the gas at the outer edges of Sextans A, determine any structure in the gas, and explore how far it extends and how the density falls off with radius.

Two other dwarf galaxies-WLM and NGC 2366 - have also been reported by Huchtmeier et al. (1981) to have extended H I with diameters that are 3-3.6 times their optical Holmberg diameters, and this gas has gone undetected by others (Hunter et al. 2001, Barnes \& De Blok 2004, Jackson et al. 2004). Both are fairly typical nearby, gas-rich dIm galaxies with modest absolute magnitudes. These two galaxies were also included in this study. Thus, we have observed three dIm galaxies with potentially extended H I disks - Sextans A, WLM, and NGC 2366 - with the GBT, and we report the results of those observations here. Basic properties of the galaxy sample are given in Table 1 .

\section{Observations and Mapping}

We used the $100 \mathrm{~m}$ single-dish GBT with the GBT Spectrometer 28-29 June 2003 to map the 21-cm H I-line emission associated with our three galaxies. The spectrometer was used in its auto-correlation total-power mode with a $12.5 \mathrm{MHz}$ bandwidth and 9-level sampling mode to yield a channel separation of $0.32 \mathrm{~km} \mathrm{~s}^{-1}$ and a full spectral coverage of $2595 \mathrm{~km} \mathrm{~s}^{-1}$. Our central velocity was the heliocentric radial velocity of the galaxy, given in Table 2. We observed 3C48 at the beginning of the observing session in order to verify pointing.

The beam-size at our frequencies is about $8.72^{\prime}$, and for most of the mapping we stepped every $2.9^{\prime}, 1 / 3$ of a beam-size, in order to produce a fully sampled map. One exception was a portion of the NGC 2366 map to the north that was begun with a step of a 1/2 beam-size. The other exception was the map of WLM which consists of an east-west strip 3 pointings wide (one offset $1 / 3$ beam width to the north and one offset $6^{\prime \prime}$ to the south) and a northsouth strip through the galaxy center. The map center, number of pointings, and final field of view of each map is given in Table 2. We began the map in the center row and worked our way north one row at a time, and then returned to the center and worked our way south. For

Sextans A, the last row of the northern part of the map was only partially completed due 
to time limitations. We used position switching for calibration and background subtraction, and our reference position was located 40-50' north or south, whichever was closer. We moved to this off position every 5 steps along the map for Sextans A and WLM and every 6 steps for NGC 2366. We integrated for 1 minute at each position, obtaining 3 spectra of $20 \mathrm{~s}$ each and expecting to reach an RMS of $0.03 \mathrm{~K}$.

The data were reduced using GBTIDL. Because we were interested only in a relatively narrow velocity range around the central velocity of the galaxies, we eliminated approximately the first and last 1000 channels in every scan in order to remove noise spikes. We calibrated each row pointing against the reference scan taken during the observations of that row. The resulting spectra showed stable, flat baselines. A few scans had curvy baselines, and those scans were flagged and not used further. The continuum on each side of the emission line was fit and subtracted to produce a baseline at zero. We smoothed along the velocity axis with a boxcar average of 5 pixels and proportionally decreased the number of channels in the final spectra. The final spectra contained 1201 channels for Sextans A and NGC 2366 and 1361 channels for WLM with channel widths of $1.61 \mathrm{~km} \mathrm{~s}^{-1}$. We used a standalone program, idlToSdfits, to convert the calibrated spectra in GBTIDL to a format that mimics radio interferometry uv data and then read those data into AIPS. Within AIPS we used SDGRD to produce map cubes with $174^{\prime \prime}$ pixels.

From the map cubes we made moment zero maps of integrated H i emission. We integrated over channels 516-579 for Sextans A, 506-594 for NGC 2366, and 610-677 for WLM. In the calibration process in GBTIDL, the GBT data are converted to $T_{a}^{*}$, antenna temperature corrected for atmospheric attenuation, radiative loss, and rearward scattering and spillover. To convert to $T_{b}$, the brightness temperature of the source, we correct for the forward scattering and spillover, $\eta_{f s s}$, and for the main beam efficiency, $\eta_{m b}$. Here we ignore forward scattering and use a typical value for $\eta_{m b}$ at $1.4 \mathrm{GHz}$ on the GBT of 0.94 so $T_{b}=1.07 \times T_{a}^{*}\left(\right.$ Braatz 2009). Then for our beam-size and frequency, $S(\mathrm{Jy})=T_{a}^{*} / 2.02$. Observations of the standard continuum source $3 \mathrm{C} 48$ yield a flux that is $6 \%$ low. However, this is within the uncertainty of $10 \%$ for $21-\mathrm{cm}$ calibration reported by Robishaw \& Heiles (2009). Thus, we expect our fluxes to be uncertain at the $10 \%$ level.

In the map cubes, the units of $\mathrm{K}_{\text {beam }}{ }^{-1}$ pixel $^{-1}$ can be converted to units of Jy by multiplying by a factor of (pixel scale) $)^{2} /$ beam-size ${ }^{2} / 2.02$, which is a factor of 0.054 . For contours on the moment zero (integrated $\mathrm{HI}$ ) maps, map units of $15.3 \mathrm{~K}^{\text {beam }}{ }^{-1} \mathrm{~km} \mathrm{~s}^{-1}$ pixel $^{-1}$ correspond to a column density of $10^{19}$ atoms $\mathrm{cm}^{-2}$.

Our flux-velocity profiles are shown in Figure1. Robishaw \& Heiles (2009) have reported inaccuracies in 21-cm line profiles measured with the GBT. They find $10 \%$ errors in the calibrated gain and some contributions from distant sidelobes. Although these problems 
may increase the uncertainties, the profile shapes agree well with those measured with the VLA.

\section{Results}

\subsection{The Extent of $\mathrm{H}_{\mathrm{I}}$}

\subsubsection{Sextans A}

We detected H I emission from Sextans A between $270 \mathrm{~km} \mathrm{~s}^{-1}$ to $372 \mathrm{~km} \mathrm{~s}^{-1}$. This is very close to the range of velocities detected by Wilcots \& Hunter (2002) and Huchtmeier et al. (1981) although their channel spacings of $2.6 \mathrm{~km} \mathrm{~s}^{-1}$ and $6.6 \mathrm{~km} \mathrm{~s}^{-1}$, respectively, were coarser than ours. A Galactic High Velocity Cloud (HVC), identified by Huchtmeier et al., is also present in the direction of Sextans A. We detect it from $110 \mathrm{~km} \mathrm{~s}^{-1}$ to $152 \mathrm{~km} \mathrm{~s}^{-1}$.

Our integrated H I map of Sextans A is shown in Figure 2 and contoured on a $V$-band image in Figure 3. Here we summed from $270.5 \mathrm{~km} \mathrm{~s}^{-1}$ to $371.9 \mathrm{~km} \mathrm{~s}^{-1}$ in the GBT data cube. The central bright object is Sextans A, and we see no other structure in the map. Our $3 \sigma$ column density limit in Figure 2 is $2 \times 10^{18}$ atoms $\mathrm{cm}^{-2}$. This is significantly better than the sensitivity limit of $7.5 \times 10^{18}$ atoms $\mathrm{cm}^{-2}$ of Wilcots \& Hunter's (2002) VLA data and the $10^{19}$ atoms $\mathrm{cm}^{-2}$ limit of Huchtmeier et al. (1981). In fact, this is only a little higher than the $3 \sigma$ limit of $1.6 \times 10^{18}$ atoms $\mathrm{cm}^{-2}$ achieved by Oosterloo et al. (2007) in ultra-deep mapping of the extended H i halo around NGC 891.

Masses and $\mathrm{H}$ I extents are given in Table 3. We calculate a total H I mass of $8.6 \times 10^{7}$ $\mathrm{M}_{\odot}$. This is only $25 \%$ more than the mass of $6.9 \times 10^{7} \mathrm{M}_{\odot}$ reported by Wilcots \& Hunter (2002), but $78 \%$ of the mass detected by Huchtmeier et al. (1981), all corrected to the distance we are using.

It is immediately clear from Figure 2 that there is no large extended skirt of $\mathrm{HI}$ around Sextans A. Figure 4 shows a column density profile constructed from measuring the azimuthally-averaged surface density of the gas in annuli. Because the beam-size is so large, the galaxy H I distribution appears largely round. We used the position angle (P.A.) and inclination given in Table 2, and the column densities are corrected for inclination. From the column density profile, we measure H I around Sextans A to a diameter of 22.1' at a column density of $10^{19}$ atoms $\mathrm{cm}^{-2}$. Corrected for the $8.7^{\prime}$ beam, this is $20.4^{\prime}$. Hunter \& Wilcots (2002) reported a diameter of $18^{\prime}$ at $7.5 \times 10^{18}$ atoms $\mathrm{cm}^{-2}$ from their VLA observations, and their surface density profile yields a diameter of $15.2^{\prime}$ at the level of $10^{19}$ atoms cm$^{-2}$ that we are using for comparison here. In short, we do not detect the extended disk of $\mathrm{H}$ I 
that Huchtmeier et al. reported in 1981. Wilcots and Hunter did not detect this gas with the VLA, not because it is extremely smooth, but because it is not there.

With Huchtmeier et al.'s H I diameter of $54^{\prime}$, the ratio of $\mathrm{H}$ I diameter to optical diameter of Sextans A was extreme. Figure 13 of Hunter (1997) compiles data on the H I extents of typical irregular galaxies. That figure is a histogram of the ratio $R_{\mathrm{HI}} / R_{\mathrm{Holm}}$ of the radius of $\mathrm{H}$ I at $10^{19}$ atoms $\mathrm{cm}^{-2}$ to the optical extent as measured by the Holmberg radius, defined at a photographic surface brightness of $26.5 \mathrm{mag} \operatorname{arcsec}^{-2}$. Most irregular galaxies, like most spirals, have a ratio around 2 . Only 6 systems were known at that time to have $R_{\mathrm{HI}} / R_{\mathrm{Holm}}$ greater than 3 , with the highest being a ratio of 7 . Sextans $\mathrm{A}$ was then thought to have the third highest ratio with a value of 5.8, with the values of $R_{\mathrm{HI}}$ and $R_{\mathrm{Holm}}$ used at that time. With our new data, we find that Sextans $\mathrm{A}$ has an $R_{\mathrm{HI}}$ at $10^{19}$ atoms $\mathrm{cm}^{-2}$ of $10.2^{\prime}$, and the ratio $R_{\mathrm{HI}} / R_{\text {Holm }}$ is 3.3. (Our $R_{\text {Holm }}$ are taken from Hunter \& Elmgreen [2006] and given in Table 1). This now puts Sextans A at the high end of the distribution of the bulk of other irregular galaxies and an $\mathrm{H}$ I extent that is not particularly abnormal.

Where could the discrepancy between the Huchtmeier et al. (1981) Effelsberg observations and the GBT observations lie? We have replicated as closely as possible the channel maps of Sextans A given in Figure 3c of Huchtmeier et al. Since their velocity resolution is lower than ours, this involved summing over the appropriate channels in the GBT data cube. These channel maps are shown in Figure 5. Each map is the average of 10 channels and is centered as closely as possible on the same velocities as Huchtmeier et al.'s channel maps. Figure 5 looks different than Figure 3c of Huchtmeier et al. The Effelsberg data detect extended H I that does not appear in our maps and the shape of Sextans A at the lowest column densities differs.

There are significant differences between the GBT observations and those done at Effelsberg that may contribute to differences in the maps. First, although the beam sizes are nearly the same, Huchtmeier et al. (1981) mapped Sextans A every beam-size (9'), whereas we mapped every $1 / 3$ of a beam $\left(2.9^{\prime}\right)$. Thus, our map is better sampled. Second, our signal-to-noise is higher, and the extended features in the Huchtmeier et al. maps occur at low signal-to-noise. Third, although the GBT does have side-lobe problems (Robishaw \& Heiles 2009), they are different from those at Effelsberg and we believe that the Effelsberg map was confusing Galactic emission with emission from Sextans A. We detect Galactic emission at lower velocities, offset about $120 \mathrm{~km} \mathrm{~s}^{-1}$. 


\subsection{2. $W L M$}

We detected H I emission from WLM between $-179 \mathrm{~km} \mathrm{~s}^{-1}$ to $-71 \mathrm{~km} \mathrm{~s}^{-1}$. Similarly, Kepley et al. (2007) detected emission from $-170 \mathrm{~km} \mathrm{~s}^{-1}$ to $-72 \mathrm{~km} \mathrm{~s}^{-1}$ and Huchtmeier et al. (1981) from $-179 \mathrm{~km} \mathrm{~s}^{-1}$ to $-64 \mathrm{~km} \mathrm{~s}^{-1}$. Our integrated H I map of WLM is shown in Figure 6 and contours are superposed on a $V$-band image in Figure 7. Because of limited telescope time, our map consists of a strip through the center along the north-south direction and along the east-west direction. This was done in order to look for extended emission along two diameters. Thus, the map is not complete and imaging artifacts are obvious. However, no extended skirt of $\mathrm{H}$ I is seen along the two map directions. Our $3 \sigma$ column density limit in Figure 6 is $9 \times 10^{17}$ atoms $\mathrm{cm}^{-2}$.

Masses and $\mathrm{H}$ I extents are given in Table 3. We calculate a total H I mass of $1.1 \times 10^{8}$ $\mathrm{M}_{\odot}$. This is $1.6 \times$ more than the mass of $7.0 \times 10^{7} \mathrm{M}_{\odot}$ reported by Kepley et al. (2007), $1.9 \times$ the mass of $5.9 \times 10^{7} \mathrm{M}_{\odot}$ detected by Huchtmeier et al. (1981), 3 times higher than the mass of Jackson et al. (2004), and 60\% higher than Barnes \& De Blok's (2004) mass —all corrected to our choice of distance for WLM. However, because of the structure of our map, our total mass is uncertain.

For WLM, rather than constructing an azimuthally-averaged column density profile since our map is incomplete, we made a simple north-south cut through the center of the galaxy. This is close to the major axis of the galaxy. This cut sums east-west over 3 pixels $\left(8.7^{\prime}\right)$, and is shown in Figure 4. The distance from the center of the galaxy is positive for the north direction and negative for south. From this cut, we see that a column density of $10^{19}$ atoms $\mathrm{cm}^{-2}$ occurs at a radius of $12.9^{\prime}$ to the south and $16.4^{\prime}$ to the north of the galaxy center. The average diameter is $29.3^{\prime}$. Corrected for the $8.7^{\prime}$ beam, this is $28^{\prime}$, which is comparable to the diameter of $29.3^{\prime}$ from the VLA observations of Kepley et al. (2007). Huchtmeier et al. (1981), on the other hand, measure a diameter of $45^{\prime}$. Our diameter gives an $R_{\mathrm{HI}} / R_{\mathrm{Holm}}$ ratio of 2.4 (using the $R_{\mathrm{Holm}}$ in Table 1), which makes it normal compared to other dwarfs.

We have replicated the channel maps of WLM given in Figure 3d of Huchtmeier et al. (1981) by summing over 10 channels in the GBT data. These channel maps are shown in Figure 8. Huchtmeier et al. detect unusually extended emission at velocities of $-163 \mathrm{~km}$ $\mathrm{s}^{-1}$ to $-229 \mathrm{~km} \mathrm{~s}^{-1}$. Figure 1 shows that we have not detected emission at those velocities. However, we do detect Galactic emission between $+50 \mathrm{~km} \mathrm{~s}^{-1}$ and $-50 \mathrm{~km} \mathrm{~s}^{-1}$. WLM is also known to be near the Magellanic Stream (Putman et al. 2003). 


\subsection{3. $N G C 2366$}

We detected H I emission from NGC 2366 from $30 \mathrm{~km} \mathrm{~s}^{-1}$ to $172 \mathrm{~km} \mathrm{~s}^{-1}$, very close to what Hunter et al. (2001) found. Our integrated H I map of NGC 2366 is shown in Figure 9. and contours are plotted on the $V$-band image in Figure 10. No extended skirt of $\mathrm{H}$ i is seen in this map. Our $3 \sigma$ column density limit in Figure 6 is $2.5 \times 10^{18}$ atoms $\mathrm{cm}^{-2}$.

Masses and $\mathrm{H}$ I extents are given in Table 3. We calculate a total H I mass of $7.4 \times 10^{8}$ $\mathrm{M}_{\odot}$. This is $7 \%$ higher than the mass of $6.9 \times 10^{8} \mathrm{M}_{\odot}$ reported by Hunter et al. (2001), $4 \%$ higher than the mass detected by Huchtmeier et al. (1981), 60\% higher than Wevers et al.'s (1986) mass, 14\% higher than Swaters et al.'s (2002) mass, and 30\% higher than Walter et al.'s (2008) mass, all corrected to the distance we are using.

Figure 4 shows a column density profile constructed from measuring the azimuthallyaveraged surface density of the gas in annuli (see Table 2). We detect $\mathrm{H}$ I at a column density of $10^{19}$ atoms $\mathrm{cm}^{-2}$ around NGC 2366 at a diameter of $23.4^{\prime}$. Corrected for the $8.7^{\prime}$ beam, this is an extent of $21.7^{\prime}$. This is similar to the extent reported by Hunter et al. (2001) from VLA observations, but less than the $30^{\prime}$ diameter reported by Huchtmeier et al. (1981). Hunter et al. report an $\mathrm{HI}$ diameter of $23.4^{\prime}$ at $1.7 \times 10^{19}$ atoms $\mathrm{cm}^{-2}$, and their surface density profile yields $25^{\prime}$ at the level of $10^{19}$ atoms $\mathrm{cm}^{-2}$ that we are using for comparison here. Our H I extent gives a ratio of $R_{\mathrm{HI}} / R_{\mathrm{Holm}}$ of 2.3 , which is normal compared to other dwarfs.

We have produced channel maps of NGC 2366 similar to those produced for Sextans A and WLM even though there is no Huchtmeier et al. (1981) figure for comparison. We summed over 10 channels in the GBT data. These channel maps are shown in Figure 11. We detect Galactic emission from $-100 \mathrm{~km} \mathrm{~s}^{-1}$ to about $25 \mathrm{~km} \mathrm{~s}^{-1}$.

\subsection{The H I Fall-Off}

What can we learn about the distribution of H I in the outer part of these galaxies? Figure 4 shows the column density H I profiles. For WLM, where we have a north-south cut, the distribution is Gaussian in shape and shows a rather rapid drop to our detection limit. For Sextans A and NGC 2366, where we have azimuthally-averaged surface densities, we also see a rather rapid drop at large radii (the logarithm of the surface density is plotted). Figure 4 of Wilcots \& Hunter (2002) also gives an H I surface density profile of Sextans A along with those of several other irregular galaxies. The outer H I of Sextans A is seen in that figure to drop off in a fashion that is similar to several other irregular galaxies and a little more or less rapidly than others. We can say that there is no abrupt truncation in the 
$\mathrm{H}$ I at a limit $\geq 10^{18}$ atoms $\mathrm{cm}^{-2}$ in Sextans $\mathrm{A}$ and at a limit $\geq 10^{19}$ atoms $\mathrm{cm}^{-2}$ in NGC 2366 and WLM.

Observations of low-z absorbers imply that galaxies could extend to as low a column density as $10^{14}$ atoms $\mathrm{cm}^{-2}$ (e.g. Penton, Stocke, \& Shull 2002) if Ly $\alpha$ absorbers are associated with individual galaxies as we know them today. However, theoretical arguments suggest that all galaxies should have sharp edges at the same column density due to the metagalactic UV background radiation (Maloney 1993). Some studies have found abrupt truncations and others have not. Van Gorkom (1993) reports that the H I in the spiral NGC 3198 is sharply truncated at $2 \times 10^{19}$ atoms $\mathrm{cm}^{-2}$, and Corbelli et al. (1989) see a steep fall-off to $2 \times 10^{18}$ atoms $\mathrm{cm}^{-2}$ in M33. On the other hand, neither Walsh et al. (1997) nor Carignan \& Purton (1998) found truncations to $1 \times 10^{19}$ atoms $\mathrm{cm}^{-2}$ around NGC 289 and DDO 154, and Portas et al. (2009) find that the H I disks in spirals drop off similarly to their detection limit. Our observations do not support disk truncation.

Limits to the H I edges are expected to come from internal sources of ionizing photons as well as from the metagalactic UV background radiation. A warp in the gas disk can allow ionizing flux escaping from the galaxy to dominate ionization of the outer H I. For example, Bland-Hawthorn et al. (1997) found ionized gas beyond the H I disk in NGC 253. Yet the emission measures of $\mathrm{H} \alpha$ and [NII] are too high for the source of ionization to be the metagalactic UV background. Instead, they argue that the source of ionization is hot young stars in the inner regions of the galaxy that see the warped outer H I disk. Certainly, all three of our galaxies have modest on-going star formation. The ionizing background radiation would produce an edge that is independent of azimuth while structure from photons escaping from within would depend on the distribution of the galaxy's gas disk (Maloney 1993). We do not see evidence for an azimuthal variation in the outer H I in our galaxies.

On the other hand, in a study of nearby spiral galaxy gas disks, Portas et al. (2009) found that the gas density drops sharply at large radii. They argue that the fall-off of $\mathrm{H}$ I begins in spiral disks at too high a column density $\left(10^{20}\right.$ atoms $\left.\mathrm{cm}^{-2}\right)$ to be due to the metagalactic UV background. Furthermore, the shape of the fall-off is similar among a wide variety of spiral galaxies. They argue instead that the shape of the fall-off of the gas density with radius is more likely due to how gas disks form, reflecting their dark matter haloes and gas accretion history. Our data do not contradict this suggestion.

Our results likely have interesting implications for the nature of the intergalactic medium on the outskirts of the Local Group. Sextans A is certainly on the outskirts and WLM might be as well. The fact that we are not seeing a truncation may imply that these galaxies have yet to encounter the intragroup radiation field in the Local Group and certainly are not yet subject to any of the gas removal processes associated with the infall of galaxies into groups. 


\section{Summary}

With GBT single-dish pointings sampling in 2.9' steps, we have constructed deep large field-of-view maps of the $\mathrm{H}$ I emission centered on three dwarf irregular galaxies. We do not detect the extended skirt of H I reported by Huchtmeier et al. (1981) in these galaxies, and conclude that Galactic emission in the field of view and side-lobe emission may have led to

confusion with the sources. We measure new diameters for the H I extents at $10^{19}$ atoms $\mathrm{cm}^{-2}$. With these new dimensions, the ratios of $\mathrm{H}$ I extent to optical extent are normal for dwarf irregular galaxies. In addition, there is no evidence for a truncation in the $\mathrm{HI}$ distribution of these galaxies $\geq 10^{19}$ atoms $\mathrm{cm}^{-2}$.

We wish to thank Dr. Glen Langston, NRAO staff member at Green Bank, for assistance with implementing our observing program and in understanding how to reduce the data with aips ++ . We also are grateful for assistance from Megan Johnson with understanding how to reduce these data in GBTIDL. ECB would like to thank Dr. Kathy Eastwood and the 2003 Research Experience for Undergraduates program at Northern Arizona University which was funded by the National Science Foundation under grant 9988007. FZ acknowledges the 2011 MIT Field Camp at Lowell Observatory and Dr. Amanda Bosh for organizing that. Funding for this work was also provided by the National Science Foundation through grant AST-0204922 to DAH.

Facilities: Green Bank Telescope

\section{REFERENCES}

Bajaja, E., Huchtmeier, W. K., \& Klein, U. 1994, A\&A, 285, 385

Barnes, D. G., \& De Blok, W. J. G. 2004, MNRAS, 351, 333

Bland-Hawthorn, J., Freeman, K. C., \& Quinn, P. J. 1997, ApJ, 490, 143

Braatz, J. 2009, "Calibration of GBT Spectral Line Data in GBTIDL v2.1," NRAO document.

Carignan, C., \& Purton, C. 1998, ApJ, 506, 125

Corbelli, E., Schneider, S. E., \& Salpeter, E. E. 1989, AJ, 97, 390

Huchtmeier, W. K., Seiradakis, J. H., \& Materne, J. 1981, A\&A, 102, 134 
Hunter, D. A. 1997, PASP, 109, 937

Hunter, D. A., \& Elmegreen, B. G. 2006, ApJS, 162, 49

Hunter, D. A., Elmegreen, B. G., \& van Woerden, H. 2001, ApJ, 556, 773

Hunter, D. A., Wilcots, E. M., van Woerden, H., Gallagher, J. S., \& Kohle, S. 1998, ApJ, 495, L47

Jackson, D. C., Skillman, E. D., Cannon, J. M., \& Côté, S. 2004, AJ, 128, 1219

Kepley, A. A., Wilcots, E. M., Hunter, D. A., \& Nordgren, T. 2007, AJ, 133, 2242

Kreckel, K., Peebles, P. J. E., van Gorkom, J. H., van de Weygaert, R., \& van der Hulst, J. M. 2011, AJ, 141, 204

Maloney, P. 1993, ApJ, 414, 41

Oosterloo, T., Fraternali, F., \& Sancisi, R. 2007, AJ, 134, 1019

Penton, S. V., Stocke, J. T., \& Shull, J. M. 2002, ApJ, 565, 720

Portas, A., Brinks, E., Usero, A., Walter, F., de Blok, W. J. G., \& Kennicutt, R. C., Jr. 2009, in The Galaxy Disk in Cosmological Context, Proceedings of IAU Symposium No. 254, eds. J. Andersen, J. Bland-Hawthorn \& B. Nordström (Cambridge: Cambridge University Press), p 52

Putman, M. E., Staveley-Smith, L., Freeman, K. C., Gibson, B. K., \& Barnes, D. G. 2003, ApJ, 586, 170

Ricotti, M., Gnedin, N. Y., \& Shull, J. M. 2001, ApJ, 560, 580

Robishaw, T., \& Heiles, C. 2009, PASP, 121, 272

Shostak, G. S., \& Skillman, E. D. 1989, A\&A, 214, 33

Swaters, R. A., van Albada, T. S., van der Hulst, J. M., \& Sancisi, R. 2002, A\&A, 390, 829

Theis, C., \& Kohle, S. 1998, in The Magellanic Clouds and Other Dwarf Galaxies, Proceedings of the Bonn/Bohcum-Graduiertenkolleg Workshop, eds T. Richtler and J. M. Braun (Aachen:Shaker Verlag), p 209

van Gorkom, J. H. 1993, in The Environment and Evolution of Galaxies, ed J. M. Shull \& H. A. Thronson (Dordrecht:Kluwer), p 345 
Walsh, W., Staneley-Smith, L., \& Oosterloo, T. 1997, AJ, 113, 1591

Walter, F., Brinks, E., de Blok, W. J. G., Bigiel, F., Kennicutt, R. C., Jr., Thornley, M. D., \& Leroy, A. 2008, AJ, 136, 2563

Wevers, B. M. H. R., van der Kruit, P. C., \& Allen, R. J. 1986, A\&AS, 66, 505

Wilcots, E. M., \& Hunter, D. A. 2002, AJ, 123, 1476

Wilcots, E. M., \& Miller, B. W. 1998, AJ, 116, 2363 

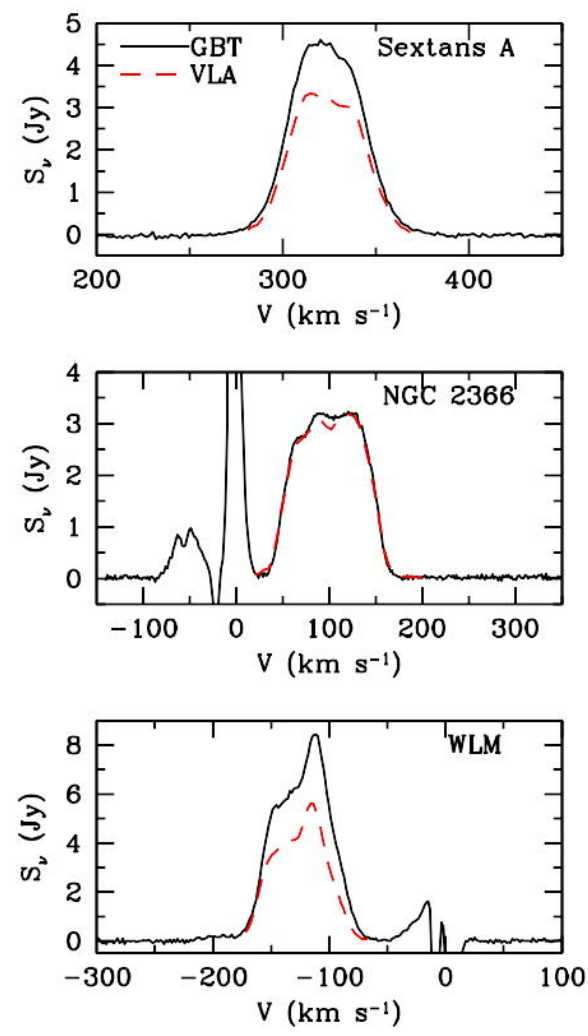

Fig. 1.- Integrated flux in individual channel maps plotted against channel velocity. Emission around zero in the NGC 2366 and WLM plots is due to the Milky Way. Profiles from VLA observations are shown as dashed lines: Sextans A data are from Wilcots \& Hunter (2002), NGC 2366 data are from Hunter et al. (2001), and WLM data are from Kepley et 


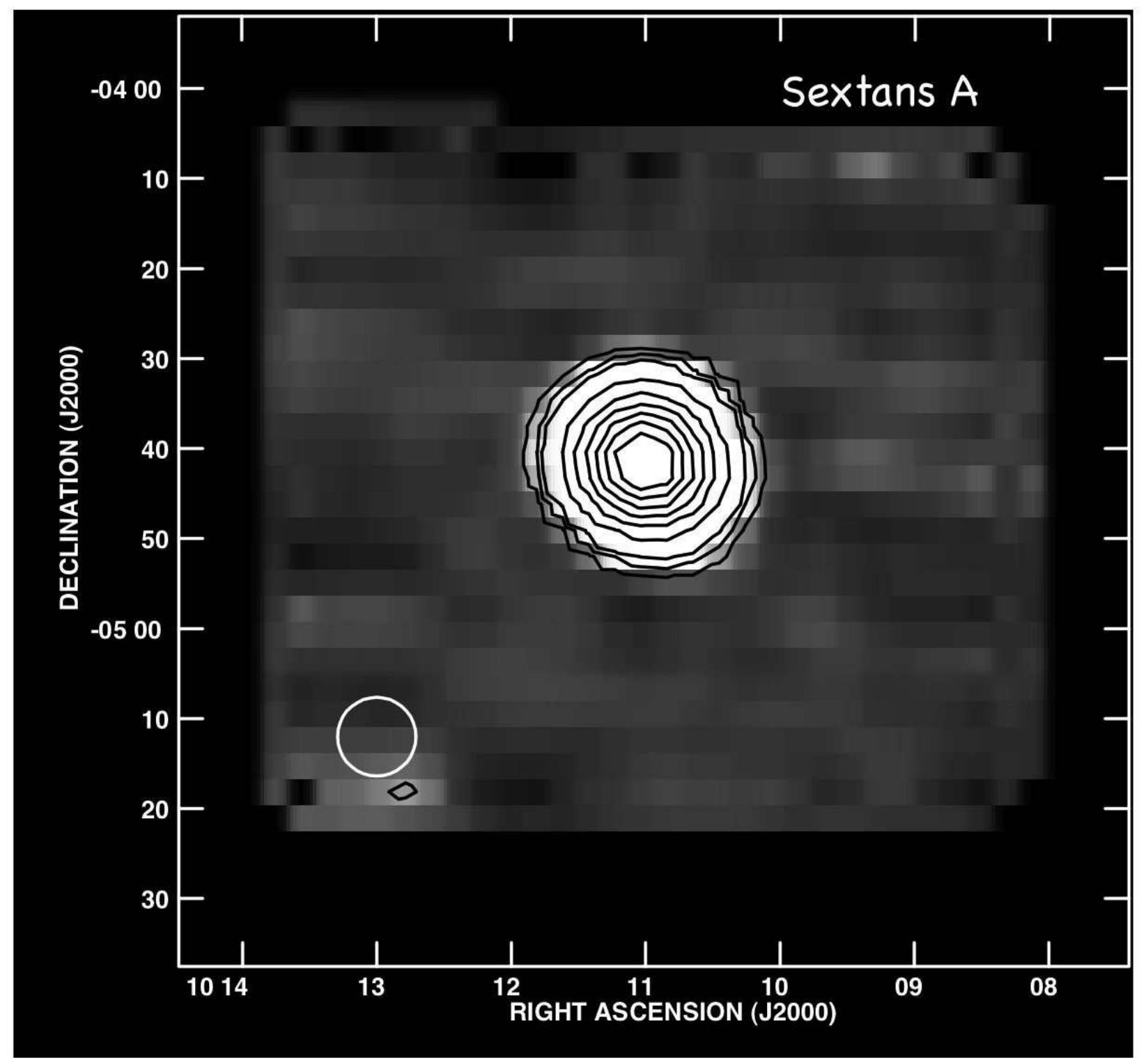

Fig. 2.- Integrated H I map of Sextans A summed from $270.5 \mathrm{~km} \mathrm{~s}^{-1}$ to $371.9 \mathrm{~km} \mathrm{~s}^{-1}$ in the GBT data cube. The image is displayed to bring out the fainter outlying structure. The circle in the bottom left corner is the FWHM of the beam: $8.72^{\prime}$ diameter. Map pixels are $2.9^{\prime}, 1 / 3$ of a beam-size. Contours are 5, 10, 15.3, 40, 70, 100, 130, 160, and $190 \mathrm{~K}$ beam $^{-1} \mathrm{~km} \mathrm{~s}^{-1}$ pixel $^{-1}$. The third contour corresponds to a column density of $10^{19} \mathrm{~cm}^{-2}$, the isodensity contour to which we have measured the HI diameters. 


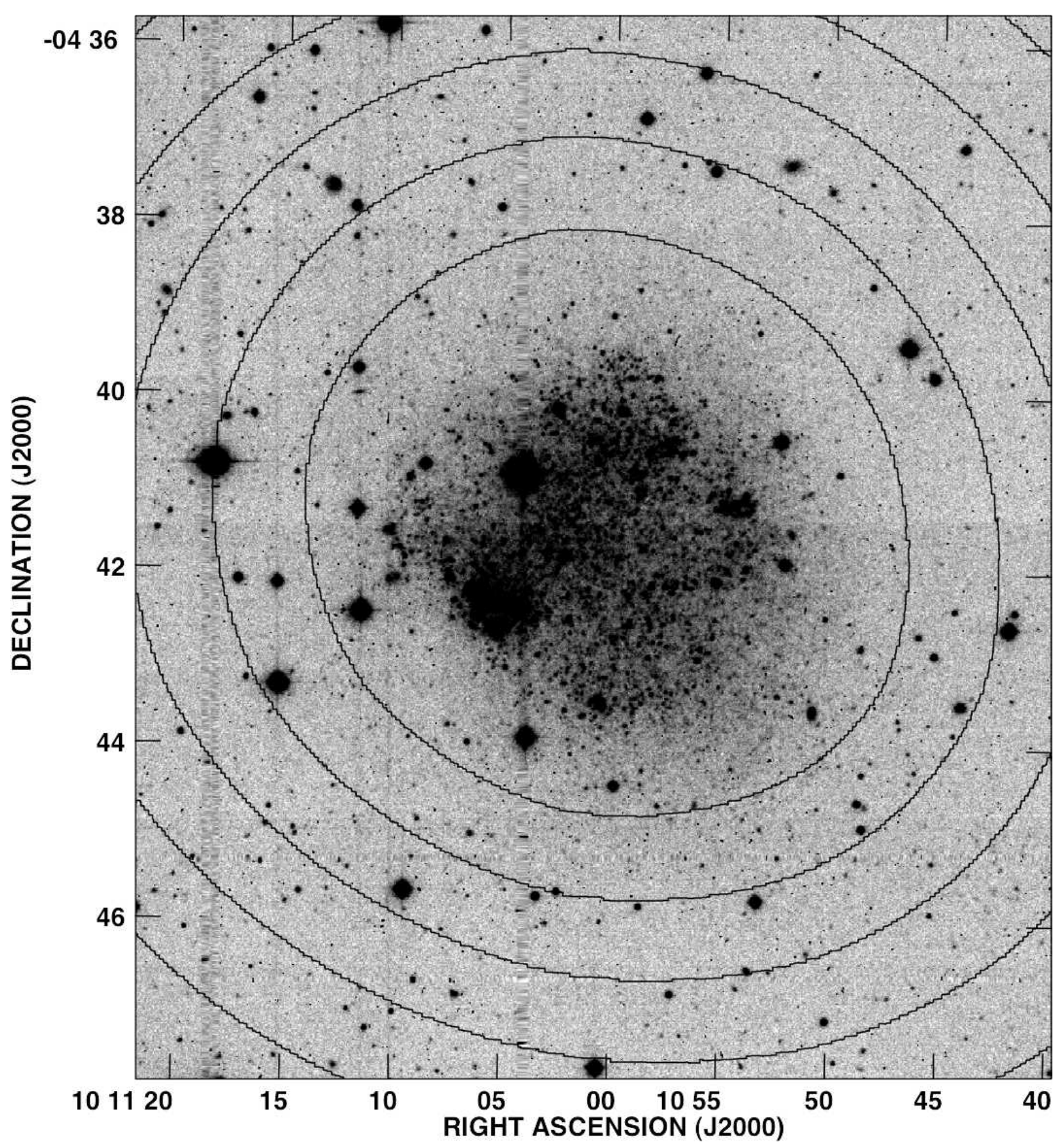

Fig. 3.- $V$-band image of Sextans A with contours from the integrated H I map superposed. Contours are the same as in Figure 2. The $V$-band image is from Hunter \& Elmegreen (2006). 

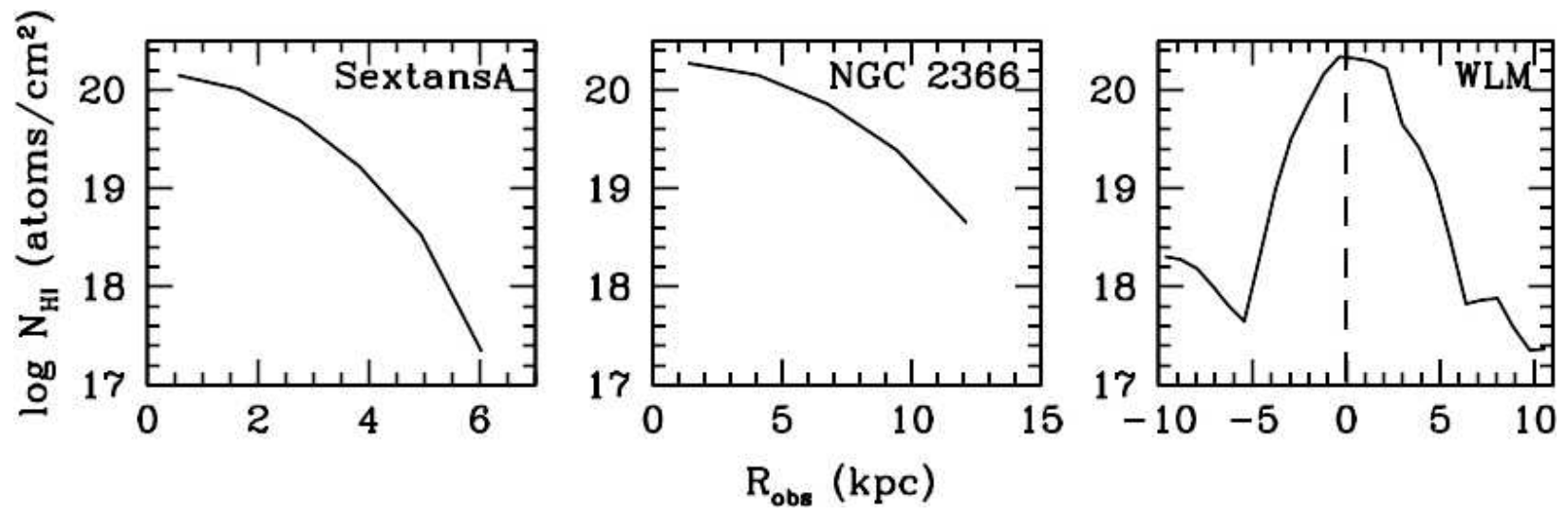

Fig. 4.- For Sextans A and NGC 2366, integrated H I mass profiles measured on the moment zero maps in annuli $174^{\prime \prime}$ wide. Surface densities are corrected for inclination of the galaxy. For WLM, we plot a north-south cut through the moment zero map, centered on the galaxy, and averaged over $8.7^{\prime}$. The vertical line at zero marks the center of the galaxy, and positive distance from that is to the north and negative to the south away from the center. $R_{o b s}$ have not been corrected for convolution with the GBT $8.7^{\prime}$ beam. 


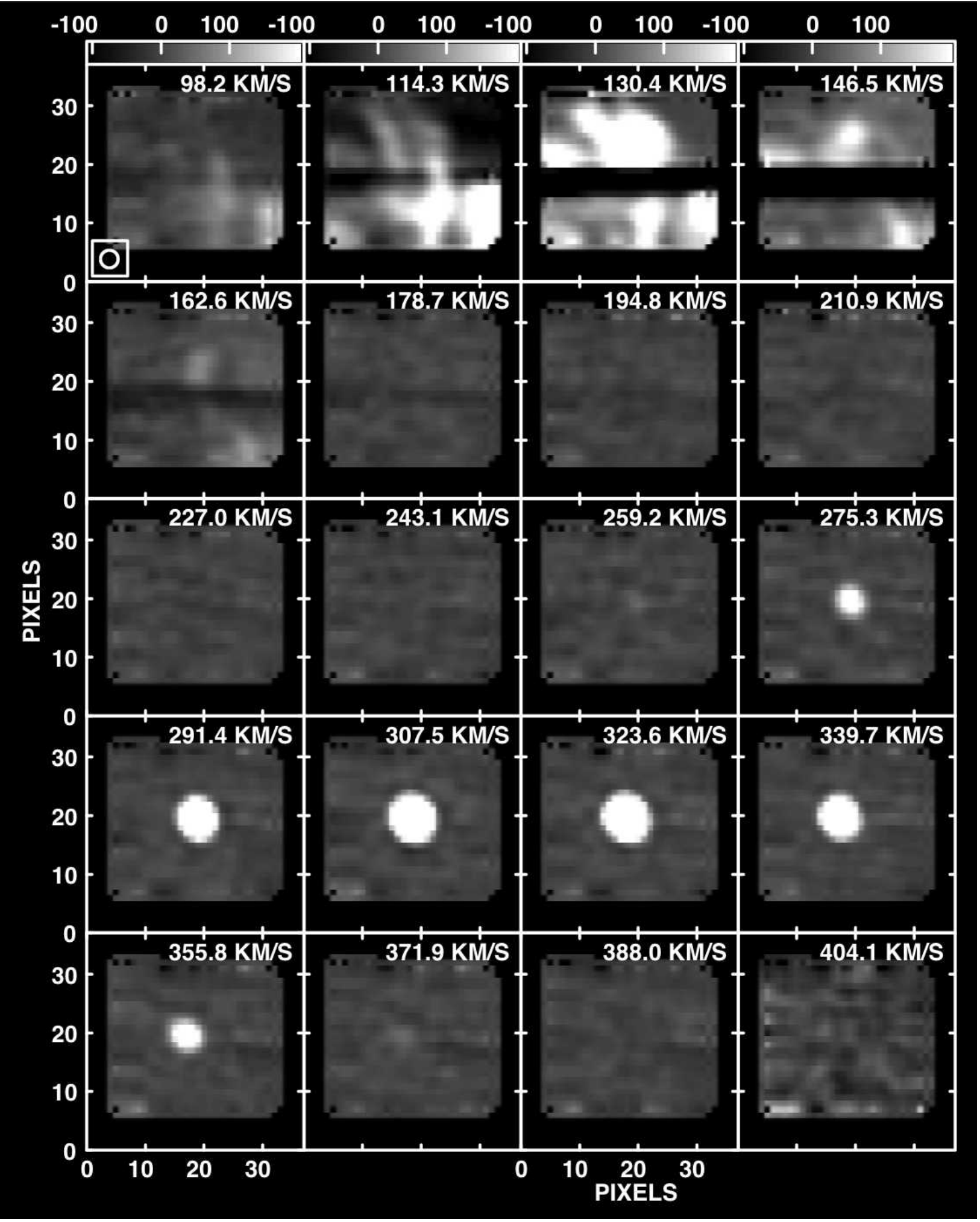

Fig. 5.- Sextans A channel maps composed from the GBT data cube to match as closely as possible the central velocity and channel spacing of channel maps shown by Huchtmeier et al. (1981) in their Figure 3c. We have summed over 10 of our channels $\left(16.1 \mathrm{~km} \mathrm{~s}^{-1}\right)$ to produce each of these channel maps. The circle in the bottom left corner of the top left panel is the FWHM of the beam: $8.72^{\prime}$. The gray scale along the top is flux in units of $\mathrm{K}$ hoom-1 nivol-1 


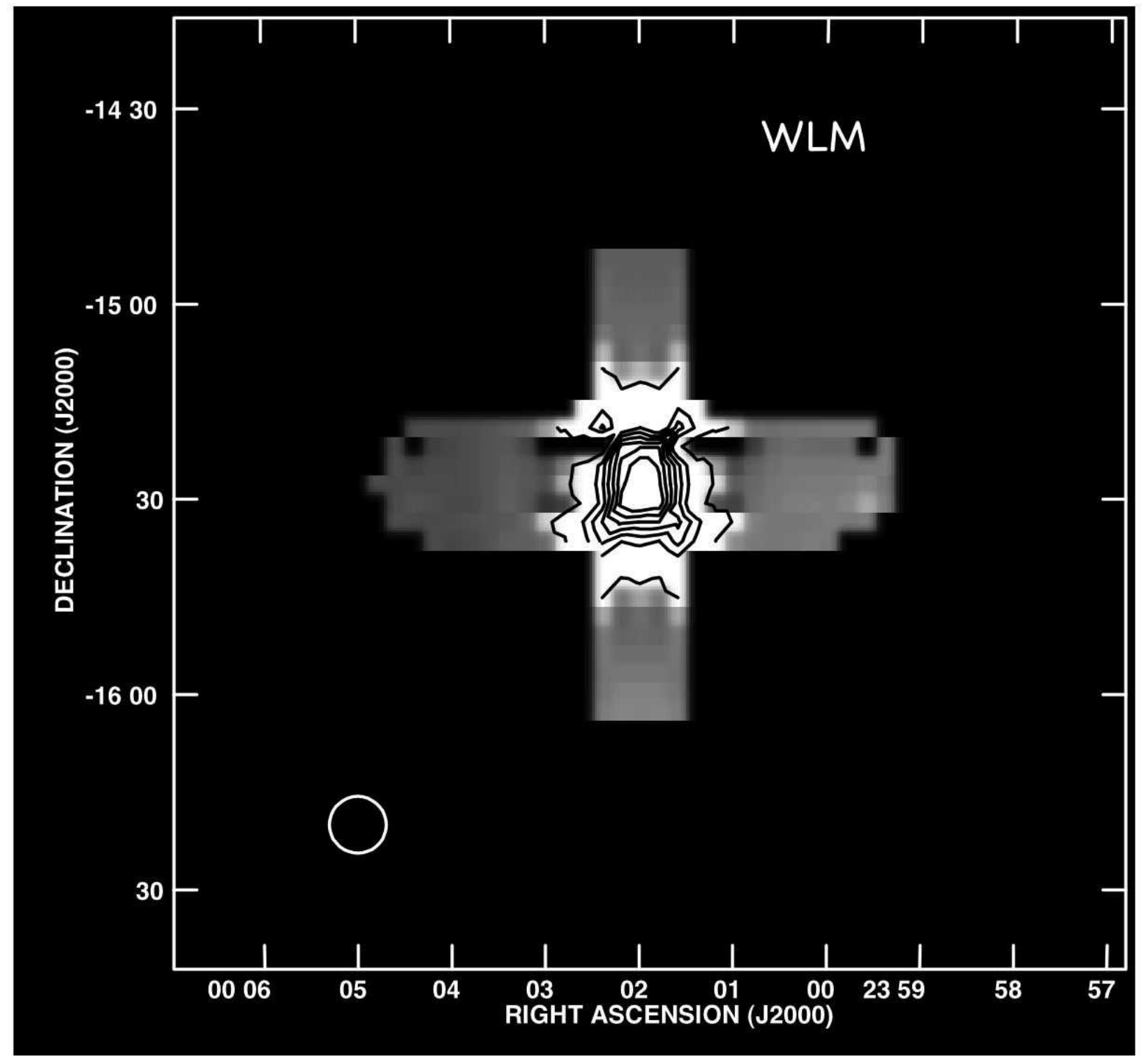

Fig. 6. - Integrated H I map of WLM summed from $-179 \mathrm{~km} \mathrm{~s}^{-1}$ to $-71 \mathrm{~km} \mathrm{~s}^{-1}$ in the GBT data cube. The image is displayed to bring out the fainter outlying structure. The circle in the bottom left corner is the FWHM of the beam: 8.72' diameter. Map pixels are 2.9', $1 / 3$ of a beam-size. Contours are 15.3, 100, 140, 180, 220, 260, and $300 \mathrm{~K} \mathrm{beam}^{-1} \mathrm{~km} \mathrm{~s}^{-1}$ pixel $^{-1}$. The lowest contour corresponds to a column density of $10^{19} \mathrm{~cm}^{-2}$, the isodensity contour to which we have measured the HI diameters. The incomplete nature of the map results in imaging artifacts. 


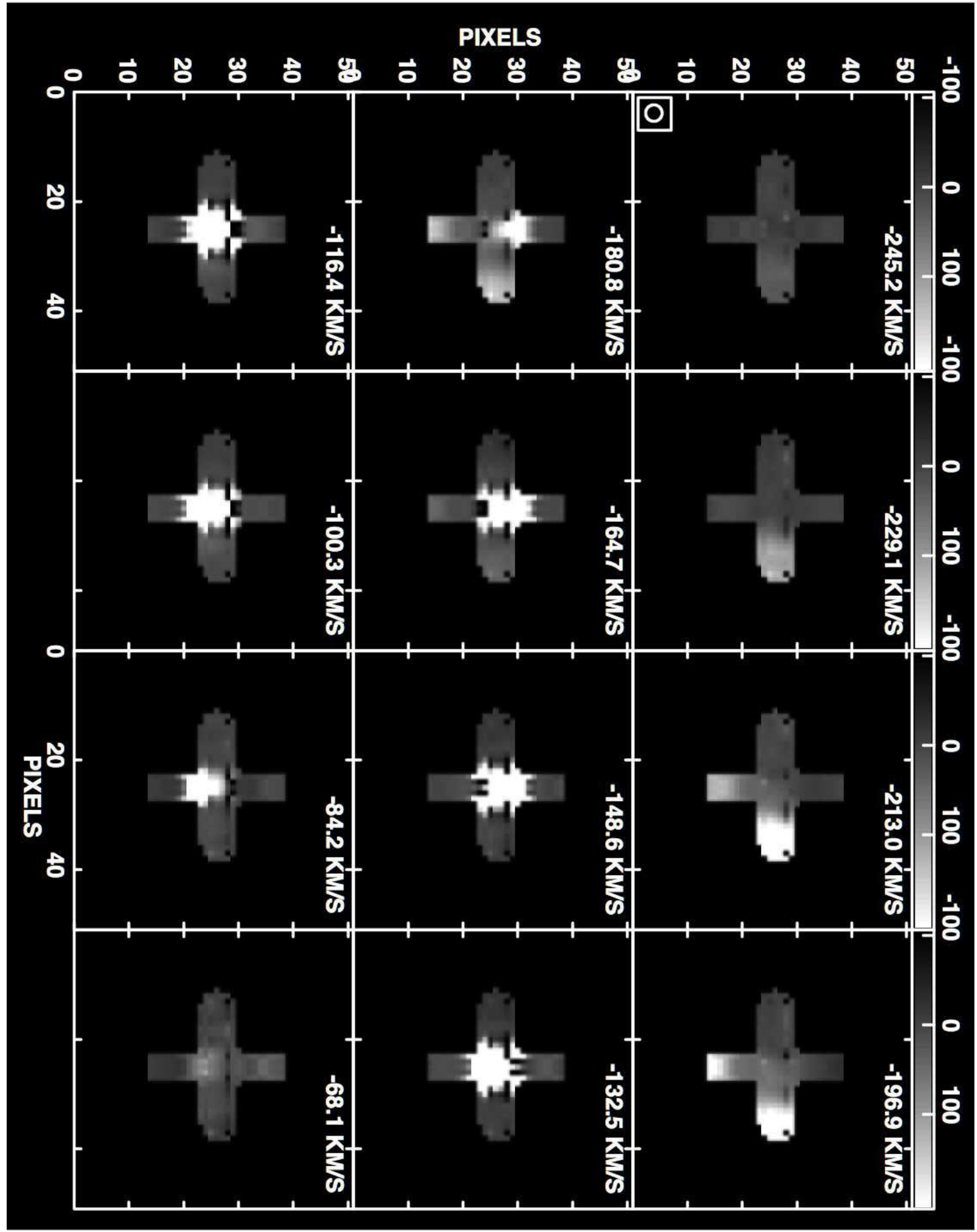

Fig. 7.- $V$-band image of WLM with contours from the integrated H I map superposed. Contours are the same as in Figure 6. The incomplete nature of the map results in imaging artifacts. The $V$-band image is from Hunter \& Elmegreen (2006). 


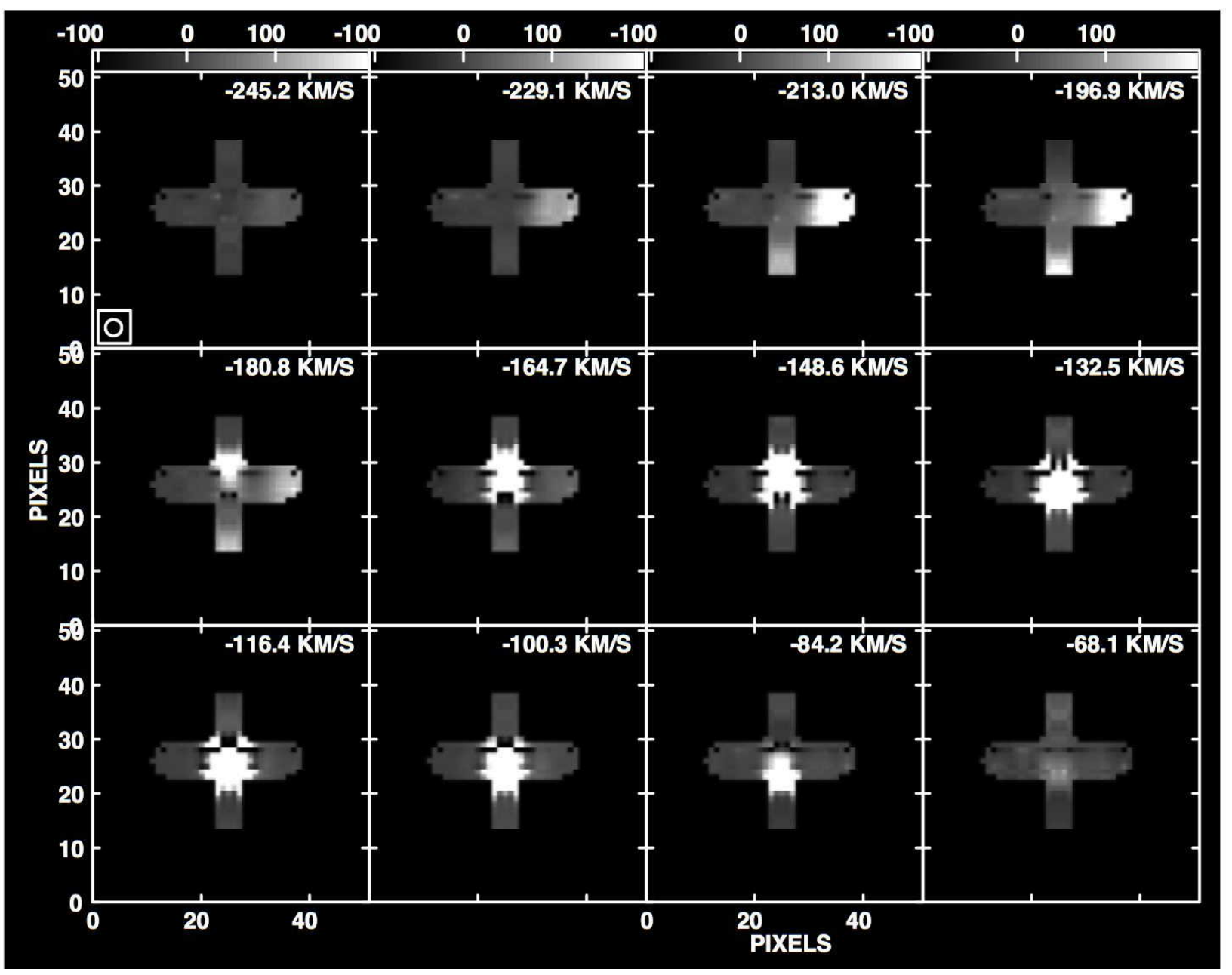

Fig. 8.- WLM channel maps composed from the GBT data cube to match as closely as possible the central velocity and channel spacing of channel maps shown by Huchtmeier et al. (1981) in their Figure 3d. We have summed over 10 of our channels $\left(16.1 \mathrm{~km} \mathrm{~s}^{-1}\right)$ to produce each of these channel maps. The circle in the bottom left corner of the top left panel is the FWHM of the beam: $8.72^{\prime}$. The gray scale along the top is flux in units of $\mathrm{K}$ beam $^{-1}$ pixel $^{-1}$. 


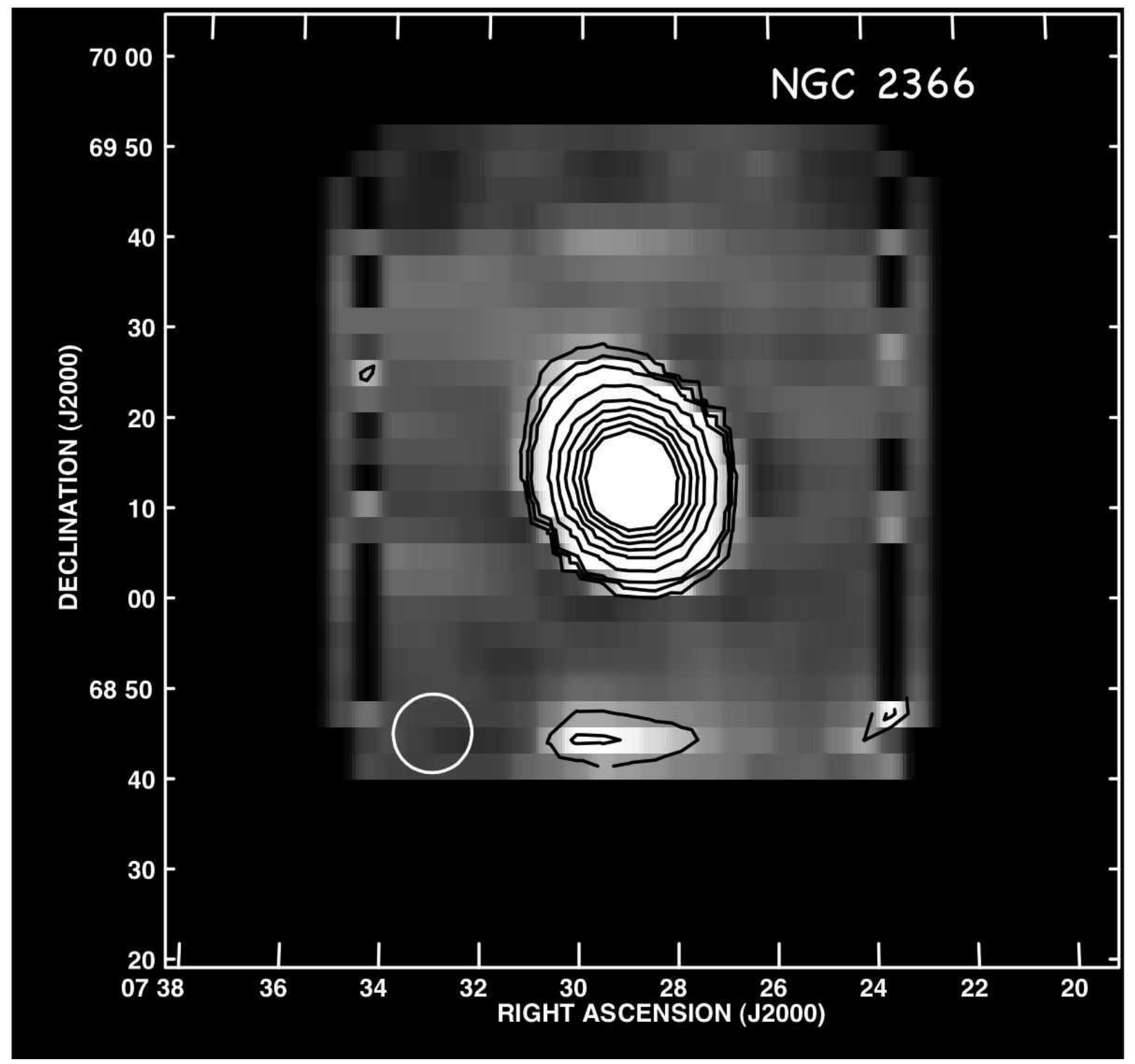

Fig. 9.- Integrated H I map of NGC 2366 summed from $30.5 \mathrm{~km} \mathrm{~s}^{-1}$ to $172.1 \mathrm{~km} \mathrm{~s}^{-1}$ in the GBT data cube. The image is displayed to bring out the fainter outlying structure. The circle in the bottom left corner is the FWHM of the beam: $8.72^{\prime}$ diameter. Map pixels are $2.9^{\prime}, 1 / 3$ of a beam-size. Contours are 5, 10, 15.3, 40, 70, 100, 130, 160, and $190 \mathrm{~K}$ beam ${ }^{-1} \mathrm{~km} \mathrm{~s}^{-1}$ pixel $^{-1}$. The third contour corresponds to a column density of $10^{19} \mathrm{~cm}^{-2}$, the isodensity contour to which we have measured the HI diameters. Examination of the data cube suggests that the emission along the bottom and right edges of the map is noise. 


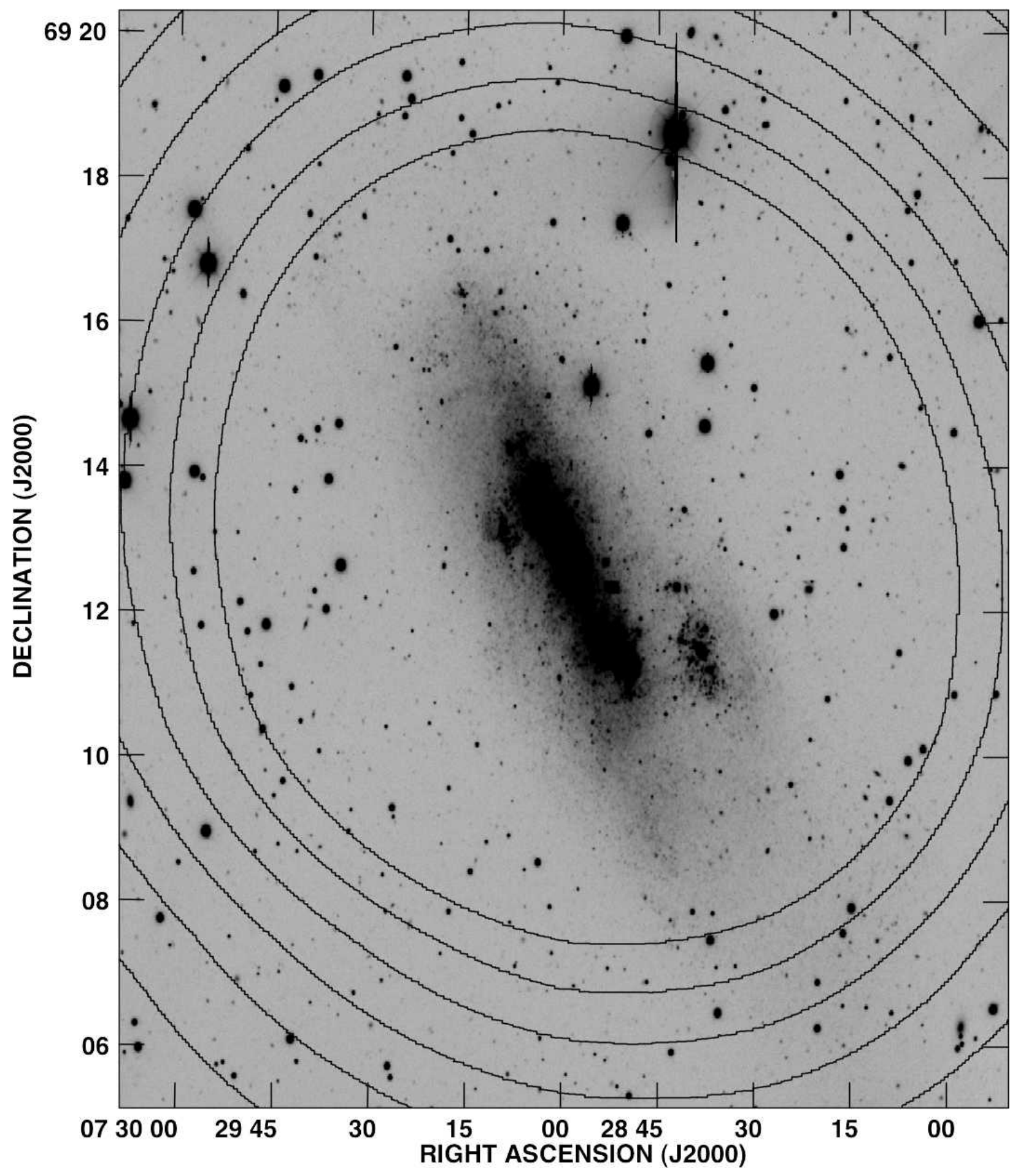

Fig. 10.- $V$-band image of NGC 2366 with contours from the integrated H I map superposed. Contours are the same as in Figure 9. The $V$-band image is from Hunter \& Elmegreen (2006). 


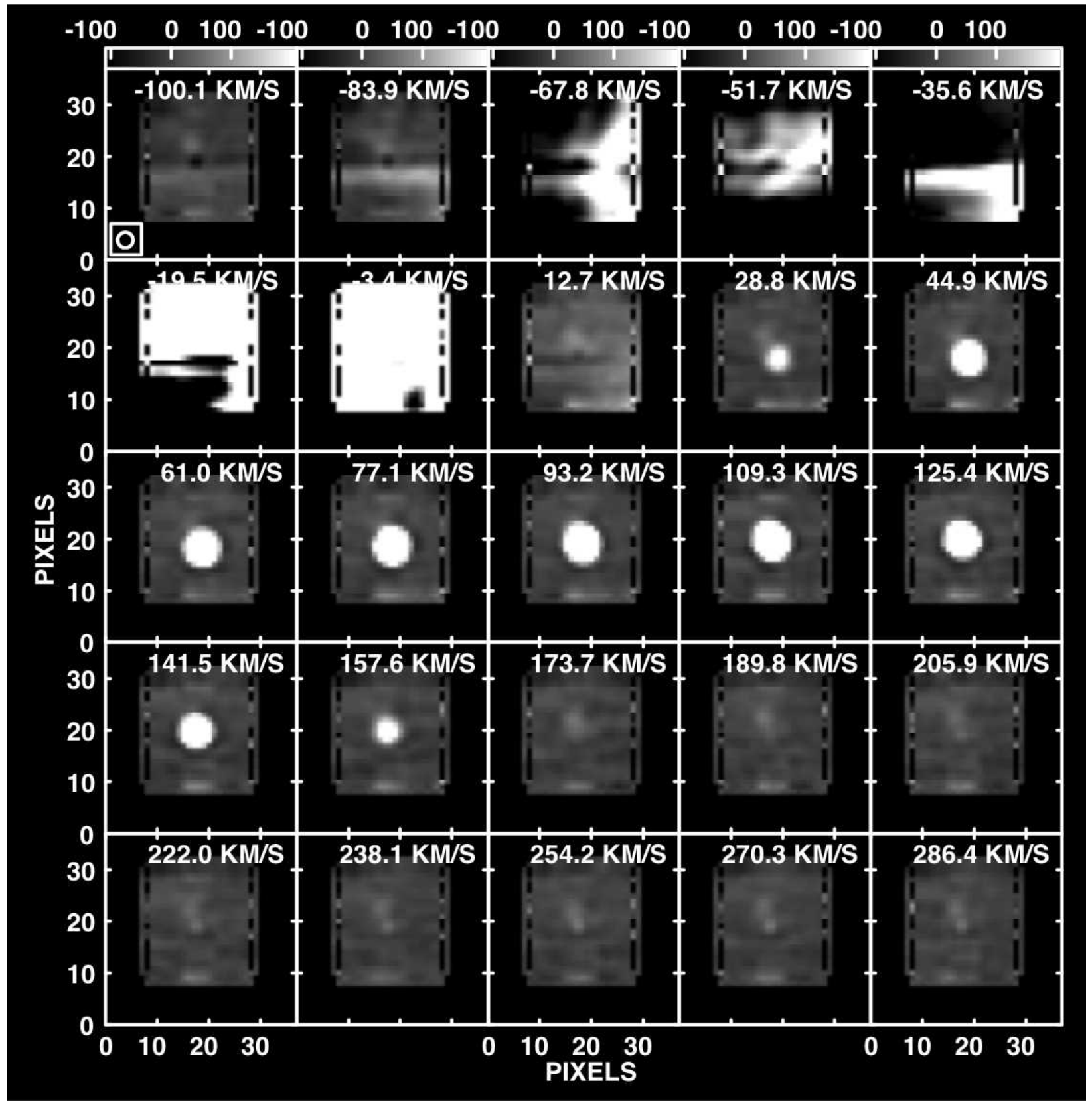

Fig. 11. - NGC 2366 channel maps composed from the GBT data cube. We have summed over 10 of our channels $\left(16.1 \mathrm{~km} \mathrm{~s}^{-1}\right)$ to produce each of these channel maps. The circle in the bottom left corner of the top left panel is the FWHM of the beam: $8.72^{\prime}$. The gray scale along the top is flux in units of $\mathrm{K}_{\text {beam }}{ }^{-1}$ pixel $^{-1}$. 


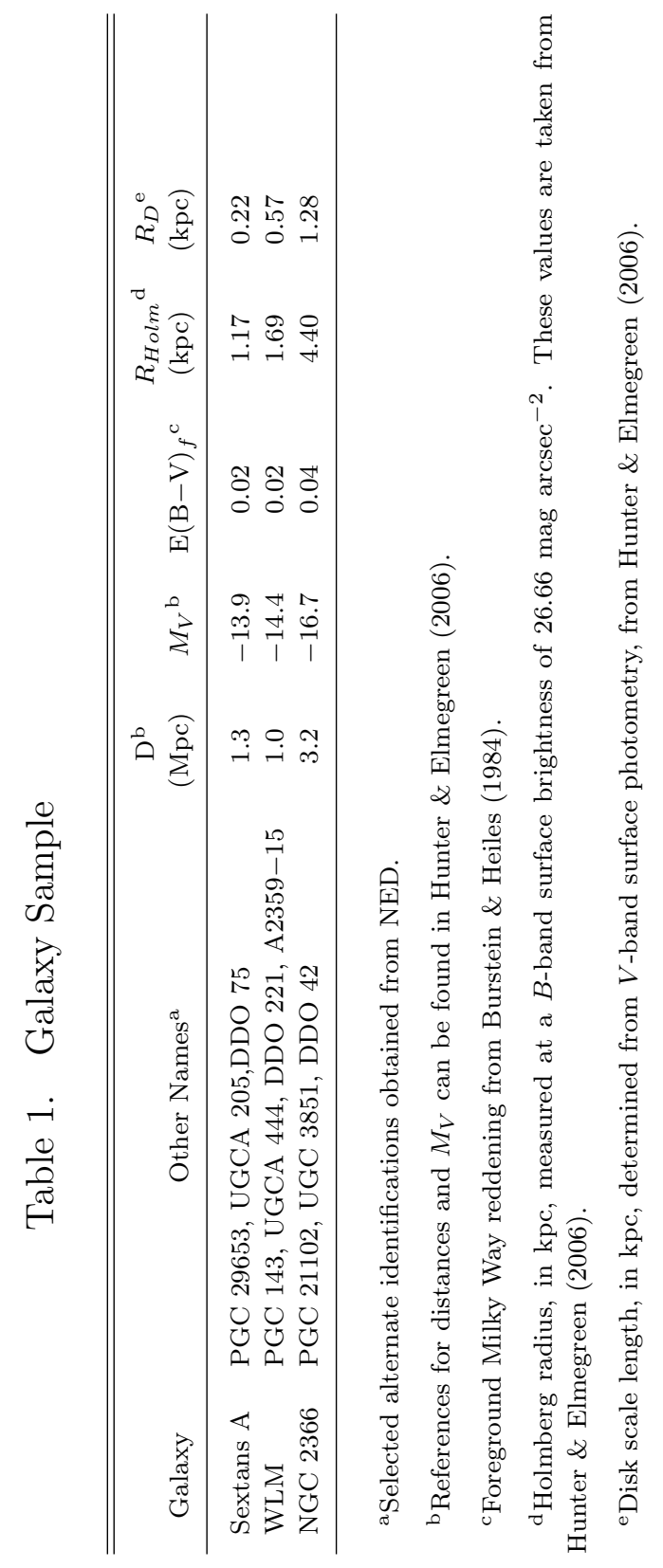




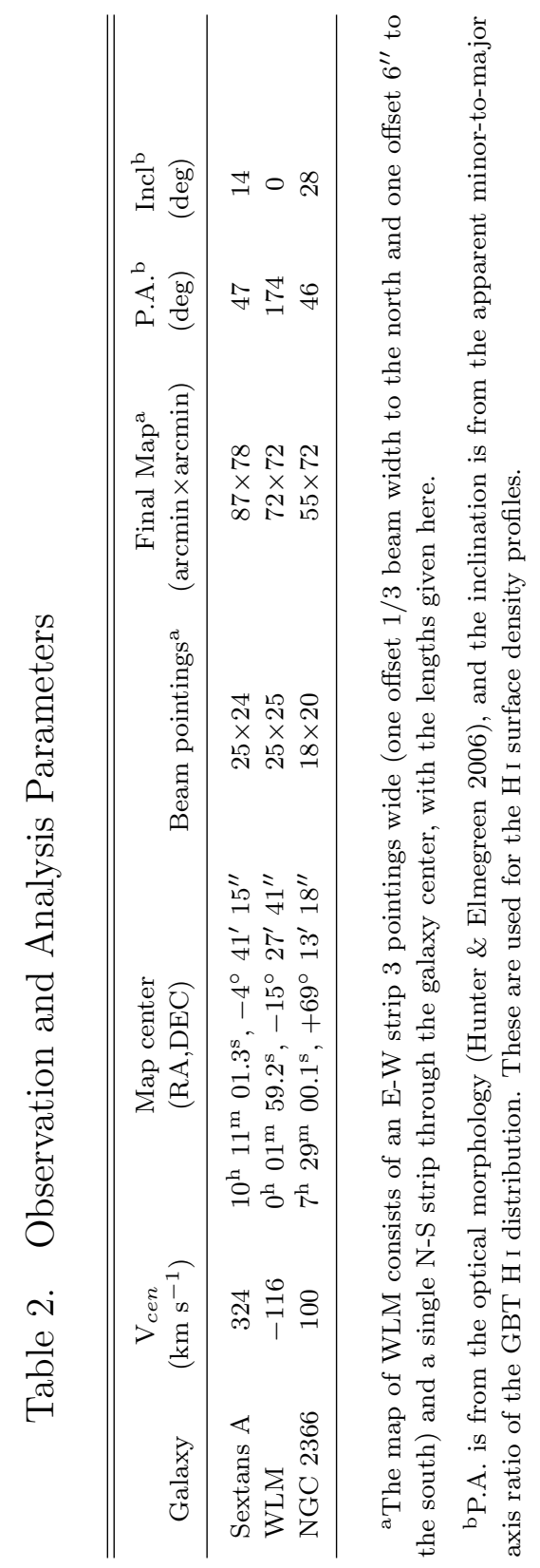




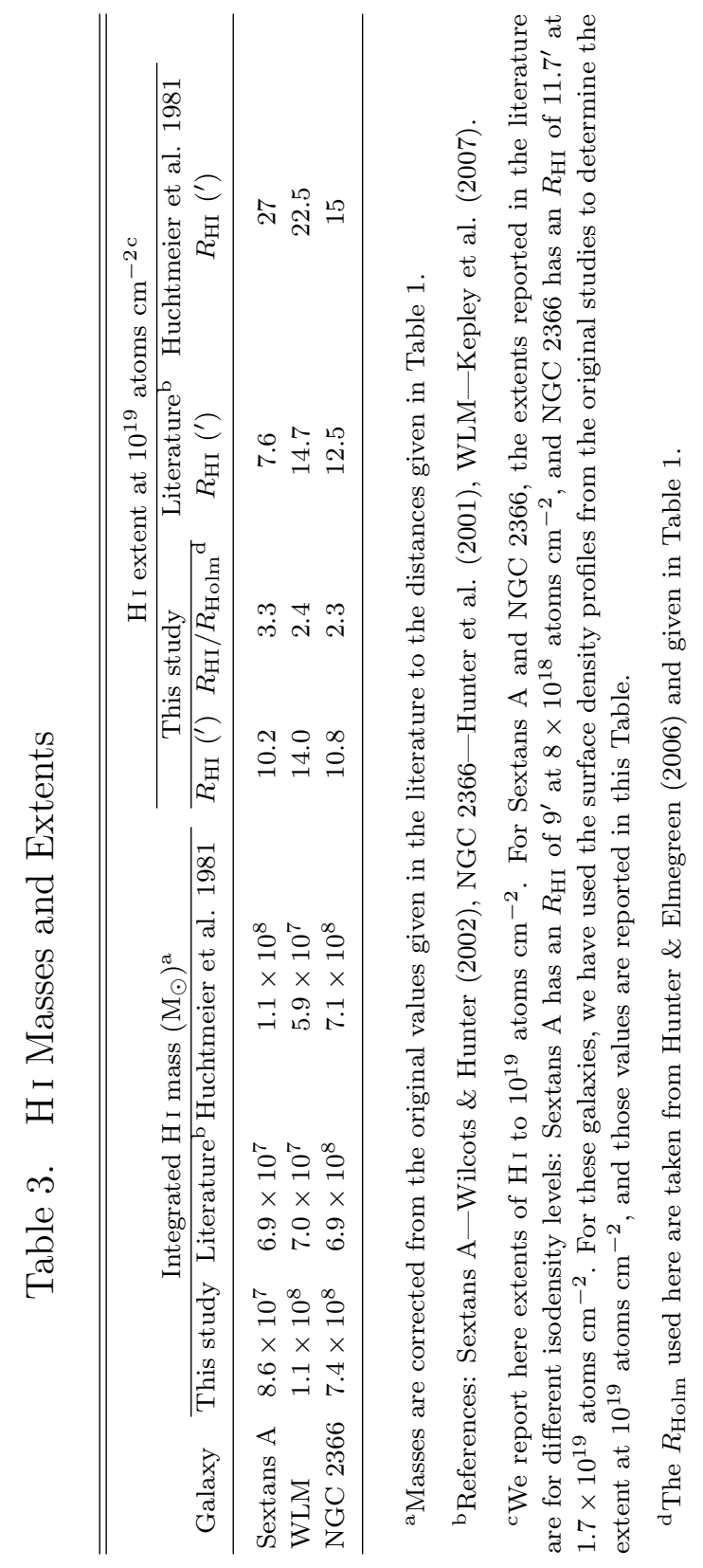

